\title{
Using all-flavor and all-sky event selections by IceCube to search for neutrino emission from the Galactic plane
}

\author{
The IceCube Collaboration ${ }^{\dagger}$ \\ $\dagger$ http://icecube.wisc.edu/collaboration/authors/icrc17_icecube \\ E-mail: kai.krings@icecube.wisc.edu
}

\begin{abstract}
The IceCube Neutrino Telescope has observed a diffuse all-flavor astrophysical neutrino flux above $30 \mathrm{TeV}$. The neutrino flux is currently compatible with an isotropic distribution; no astronomical counterparts have been identified yet. Here, we propose a binned forward-folding likelihood fit of the available neutrino data to search for integrated emission in the Galactic plane. We use two independent neutrino samples, one consisting of all-sky all-flavor events with deposited energies larger than $1 \mathrm{TeV}$ whose interaction vertex is contained in the detector's fiducial volume, the other of up-going muon neutrinos; both samples are integrated over a time period of six years. By performing a joint analysis of through-going and starting events, degeneracies between Galactic and extra-galactic neutrinos can be resolved and uncertainties in the atmospheric neutrino fluxes are better controlled. We present preliminary sensitivities for different models of neutrino emission from the Galactic plane.
\end{abstract}

\footnotetext{
Corresponding authors: Kai Krings ${ }^{* 1}$

${ }^{1}$ Physik-Department, Technische Universität München, James-Franck-Str. 1, D-85748 Garching, Germany
}

35th International Cosmic Ray Conference - ICRC2017

10-20 July, 2017

Bexco, Busan, Korea

${ }^{*}$ Speaker. 


\section{Introduction}

IceCube has observed neutrinos of extra-terrestrial origin using two independent detection channels: high-energy cascade and track-like events whose interaction vertices are contained inside the detector's fiducial volume and through-going muon tracks entering the detector from below. Cascade-like events are induced by neutrinos of all flavors: the measured light from the evolving cascade of secondary particles leaves a spherical imprint in the detector due to photon scattering, which challenges the reconstruction of the incoming neutrino's direction; current algorithms achieve a median angular resolution of about $15^{\circ}$. On the other hand, track-like events are primarily produced in charge-current muon neutrino interactions and the emitted light along the trajectory of the outgoing muon functions as a lever arm that allows reconstruction of the incoming neutrino detection with an uncertainty of better than $2^{\circ}$. Due to their containment in the detector, the energy resolution of starting events is on the order of $20 \%$; through-going muon tracks in contrast deposit only part of the initial neutrino energy in the detector, which gives a lower limit on the neutrino energy itself. Starting cascade and track-like events are selected from all directions based on a veto-driven event selection, while through-going muon tracks are only selected from the northern sky; the latter are free of background from atmospheric muons, after application of all selection criteria; see section 4 .

The two independent measurements of the astrophysical neutrino flux are in agreement above $200 \mathrm{TeV}$ with a single isotropic power-law flux, which has a spectral index close to 2 [1]. Our current interpretation is that this part of the neutrino energy spectrum is of extra-galactic origin. Assuming the power-law to be unbroken over the entire energy range, the individual best-fit spectral shapes are in tension at the $2 \sigma$ level [1]. We will follow the assumption that IceCube is measuring the superposition of a hard extra-galactic neutrino flux and a softer second astrophysical flux component of Galactic origin, which motivates the search presented here for an integrated neutrino flux along the Galactic plane, using a binned forward-folding likelihood template fit.

Galactic neutrinos are expected from the decay of light mesons produced in interactions of cosmic-rays with the matter in the Galaxy or they could be produced in the direct vicinity of potential Galactic cosmic-ray accelerators. A search for a Galactic neutrino flux has already been performed using 7 years of through-going muon track data only [2]. Because of the good angular resolution, through-going muon tracks can easily probe the northern sky for an anisotropy in the arrival direction of neutrinos due to the Galactic plane. Moreover, they give a precise measurement of the extra-galactic part of the astrophysical neutrino flux at higher energies and constrain the conventional and prompt (not observed yet) atmospheric neutrino fluxes at lower energies. A discovery with this detection channel alone is however not feasible due to the limited sky coverage of the analysis and the overwhelming background of atmospheric neutrinos below $100 \mathrm{TeV}$. That said, a joint analysis with the starting event selection is the obvious next step, taking advantage of the positive features of both detection channels: good angular resolution and large statistics in throughgoing muon tracks to constrain the extra-galactic and atmospheric neutrino fluxes, and good energy resolution, full-sky coverage, and low energy threshold of starting events to disentangle Galactic and extra-galactic neutrino flux. 


\section{Analysis method}

The binned forward-folding likelihood template fit is based on a simple Poisson likelihood ${ }^{1}$,

$$
-\log (\mathscr{L})=\sum_{i} \mu_{i}(\vec{\theta}, \vec{\xi})-d_{i} \log \left(\mu_{i}(\vec{\theta}, \vec{\xi})\right)+\cdots+\sum_{k} f\left(\xi_{k}\right),
$$

which is summed over the bins $i$ of the binned experimental data $d_{i}$ and the sum $\mu_{i}$ of all binned Monte Carlo (MC) flux templates listed in Table 1. Each template describes the number of lepton

\begin{tabular}{ll} 
Lepton flux template & Lepton flux model \\
\hline Atmospheric muons & GaisserH4a [3] \\
Conventional atmospheric neutrinos & HKKM06 [4] \\
Prompt atmospheric neutrinos & ERS [5] \\
Extra-galactic neutrinos & isotropic, unbroken power-law \\
Galactic plane neutrinos & Fermi-LAT, KRA- $\gamma$
\end{tabular}

Table 1: Lepton flux templates that enter the forward-folding likelihood template fit. For more details about the Galactic plane models, see section 3. The conventional atmospheric neutrino flux model is re-weighted with respect to the cosmic-ray knee.

events expected from the modeled lepton fluxes of atmospheric or astrophysical origin that describe the experimental data. The log-likelihood function is minimized numerically with respect to the physics parameters $\vec{\theta}$ and nuisance parameters $\vec{\xi}$ upon which the MC templates depend. The physics parameters we are interested in are the Galactic and extra-galactic flux normalizations and spectral indices. The nuisance parameters include all other flux normalizations, the primary cosmic-ray (CR) spectral index relative to the model prediction, which enters both atmospheric neutrino flux components, and the relative contribution of neutrinos from kaon decays to the conventional atmospheric neutrino flux. The primary CR spectral index and the kaon fraction affect the energy spectrum of atmospheric neutrinos; additionally, the kaon fraction changes the ratio of electron to muon neutrinos and thus the ratio of cascade to track-like events. They are both implemented in a way that they do not change the total numbers of expected conventional and prompt neutrino events in order to avoid degeneracies with the atmospheric flux normalizations. Gaussian penalty terms,

$$
f(\xi)=\frac{\left(\xi-\xi_{o}\right)^{2}}{2 \sigma_{\xi}^{2}}
$$

are incorporated in the log-likelihood function to arbitrarily increase the negative log-likelihood when the nuisance parameter $\xi$ differs too much from its mean expectation $\xi_{0}$ relative to its uncertainty $\sigma_{\xi}$. For the primary CR spectral index and the kaon fraction, we assume uncertainties of 0.05 and $10 \%$, respectively; all other nuisance parameters are completely free to float. In a later stage, we will also include detector-related nuisance parameters like the optical efficiency of the digital optical modules and the optical properties of the South Pole ice.

Experimental data and MC flux templates are binned in estimated energy, declination, and right ascension. Starting events are also separated into cascade and track-like subsets.

\footnotetext{
${ }^{1}$ Constant terms are not shown.
} 


\section{Galactic plane models}

In this analysis, we focus on the predicted diffuse neutrino flux from the decay of light mesons produced in interactions of cosmic rays with the matter in the Galaxy. Two models are taken into account that are described in the following.

\subsection{Fermi-LAT model}

This is our more generic benchmark model based on Fermi-LAT's observation of diffuse gamma-ray emission from the Galaxy [6]. We take the $\pi^{0}$-component of the fit to the gammaray data as a tracer for the intensity of neutrino emission along the Galactic plane and normalize it to construct a spatial probability density function for our neutrino flux model. For the energy part, we assume an unbroken power-law. Figure 1 shows the expected differential neutrino flux at $10 \mathrm{TeV}$ for an spectral index of 2.5.
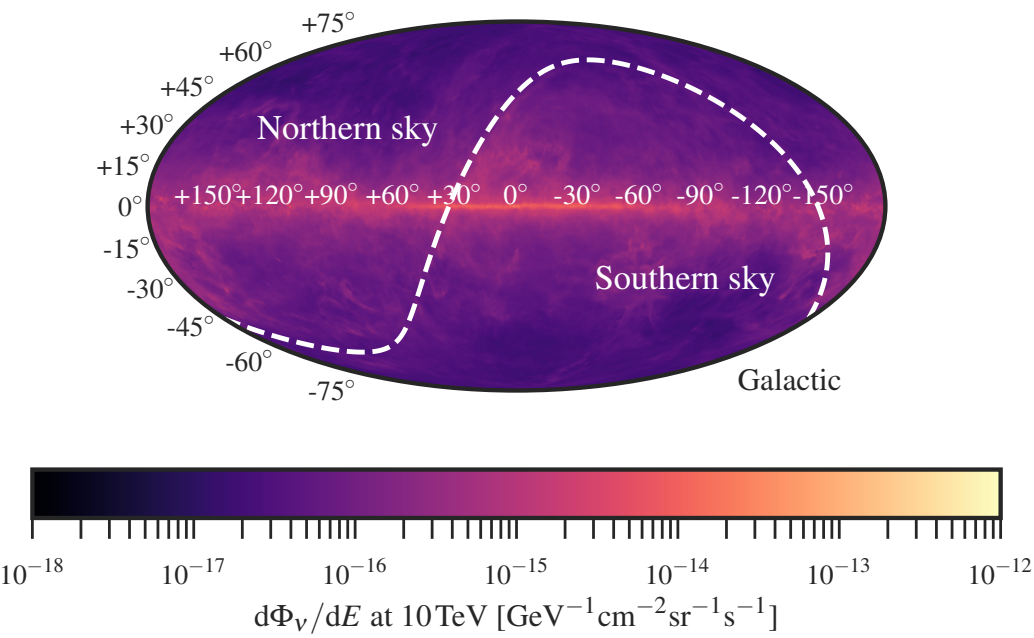

Figure 1: Fermi-LAT model for a spectral index of 2.5: differential all-flavor neutrino flux in Galactic coordinates, assuming a neutrino flavor ratio of 1:1:1. The model is normalized with respect to the KRA- $\gamma$ model.

\subsection{KRA- $\gamma$ model}

This model is based on the work of Gaggero et al. and was developed to describe the large gamma-ray flux observed by Milagro at $\mathrm{TeV}$ energies and the measured Fermi-LAT gamma-ray flux from the inner Galaxy that is systematically underestimated by the conventional diffuse gammaray emission models [7]. The predicted differential neutrino flux defines our best-case scenario. As shown in Fig. 2, the expected neutrino flux is more concentrated towards the inner Galaxy and the Galactic Center than the benchmark Fermi-LAT model. The KRA- $\gamma$ model incorporates the observed hardening of the cosmic-ray spectral slope above $250 \mathrm{GeV}$ per nucleon, which leads to the neutrino spectral slope presented in Fig. 5. 

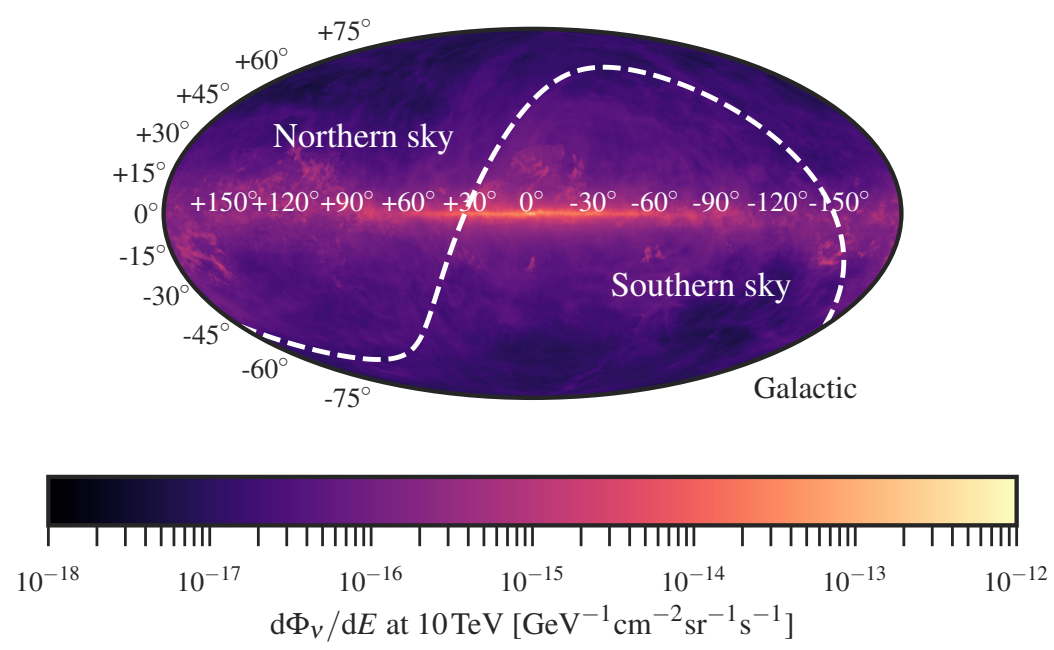

Figure 2: KRA- $\gamma$ model for an exponential high-energy cosmic-ray cutoff at $50 \mathrm{PeV}$ per nucleon: differential all-flavor neutrino flux in Galactic coordinates, assuming a neutrino flavor ratio of 1:1:1.

\section{IceCube event selections}

As motivated in section 1, two types of event topologies will be used in this analysis: all-sky and all-flavor starting events and through-going muon tracks originating from the northern sky. Both samples are integrated over a livetime of 6 years, starting from the 2010 detector run in its 79 string configuration.

\subsection{Starting events}

The selection of cascade and track-like events starting inside the detector described in [8] is the updated version of the one published in [9]. It is completely based on straight cuts, using the outer layers of IceCube as an active veto against incoming atmospheric muons. In comparison to the high-energy starting event selection, which is a subsample of this selection, the low-energy threshold is lowered down to about $1-10 \mathrm{TeV}$. The muon veto acts also as a veto against atmospheric neutrinos that accompany the incoming muon. We differentiate between correlated and uncorrelated veto where muon and neutrino either do or do not originate from the same interaction vertex in the atmospheric air shower. A refined estimation of the veto probability is still under study; at this stage, were are using the parametrization obtained in [10] with veto threshold energies for both conventional and prompt atmospheric neutrinos of 100 and $1250 \mathrm{GeV}$ below and above a total deposited charge in the detector of 6000 photoelectrons, respectively.

\subsection{Through-going muon tracks}

Well-reconstructed through-going muon tracks are selected based on Boosted Decision Trees, as described in [11]; the background of mis-reconstructed atmospheric muons is efficiently removed and a neutrino purity of $99.7 \%$ is achieved. The experimental data, which was taken between 2008 and 2014, consists of about 350000 neutrino-induced events; it is dominated by conventional atmospheric neutrinos, but sensitive to neutrinos of astrophysical origin above $200 \mathrm{TeV}$. 
The best-fit isotropic astrophysical neutrino flux per flavor is

$$
\frac{\mathrm{d} \Phi_{v+\bar{v}}}{\mathrm{~d} E_{V}}=0.9_{-0.27}^{+0.30} \times 10^{-18} \mathrm{GeV}^{-1} \mathrm{~cm}^{-2} \mathrm{~s}^{-1} \mathrm{sr}^{-1}\left(\frac{E_{V}}{100 \mathrm{TeV}}\right)^{-2.13 \pm 0.13},
$$

and no prompt atmospheric neutrinos were observed, yielding an upper limit of 1.06 times the flux prediction by Enberg et al. These results are used as the seed values for the extra-galactic and prompt atmospheric neutrino flux fit components in our analysis and as the input for the calculation of sensitivities and discovery potentials.

\section{Sensitivities and discovery potentials}

In the following we will focus only on starting events; through-going muon tracks are not included in the analysis framework yet.

Figure 3 shows the number of expected starting cascade and track-like events of extra-galactic and Galactic origin binned in reconstructed declination and right ascension, assuming the flux in Eq. (4.1) and the KRA- $\gamma$ model, respectively. Reconstruction algorithms dedicated to the individual event topologies are used and the binning corresponds to the one used in the likelihood fit. The bin size has been chosen according to the angular resolution. In total, we expect about 94 events of extra-galactic origin, 36 events of Galactic origin, and twice as many cascade as track-like events in 6 years of data; the latter are mostly up-going. The most Galactic neutrinos are expected in the bins containing the Galactic center.
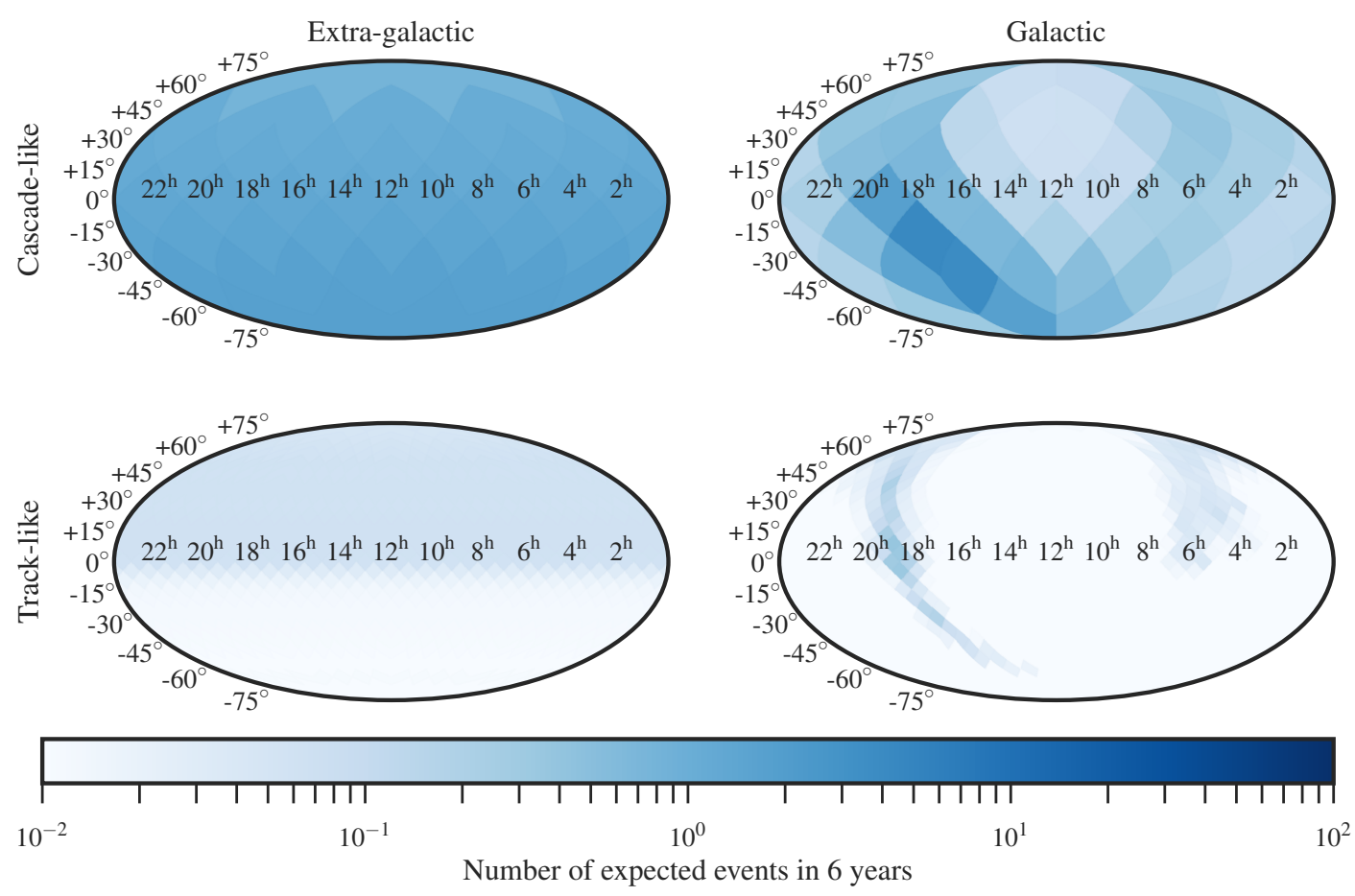

Figure 3: Expectation for extra-galactic and Galactic (KRA- $\gamma$ ) neutrinos in equatorial coordinates. The pixels have a resolution of about $30^{\circ}$ and $7^{\circ}$ for starting cascade and track-like events, respectively. 
Taking the negative log-likelihood function (2.1), we define the test statistic

$$
t=-2 \times \log \left(\frac{\mathscr{L}\left(\hat{c}_{\mathrm{GP}}, \hat{\gamma}_{\mathrm{GP}}, \ldots\right)}{\mathscr{L}\left(c_{\mathrm{GP}}=0, \ldots\right)}\right)
$$

in order to test the extra-galactic plus Galactic neutrino flux hypothesis against the single isotropic astrophysical flux hypothesis (null-hypothesis). The test statistic depends on the best-fit Galactic flux normalization $\hat{c}_{\mathrm{GP}}$, the spectral index $\hat{\gamma}_{\mathrm{GP}}$, and the nuisance parameters described in section 2 . For the sensitivity and discovery potential estimation, we assume the null-hypothesis, create an Asimov dataset [12], and scan the Galactic flux normalization until we can exclude the null-hypothesis at $90 \%$ and $3 \sigma$ confidence level (CL), respectively, using a $\chi^{2}$-distribution with 1 degree of freedom for the background test statistic distribution.

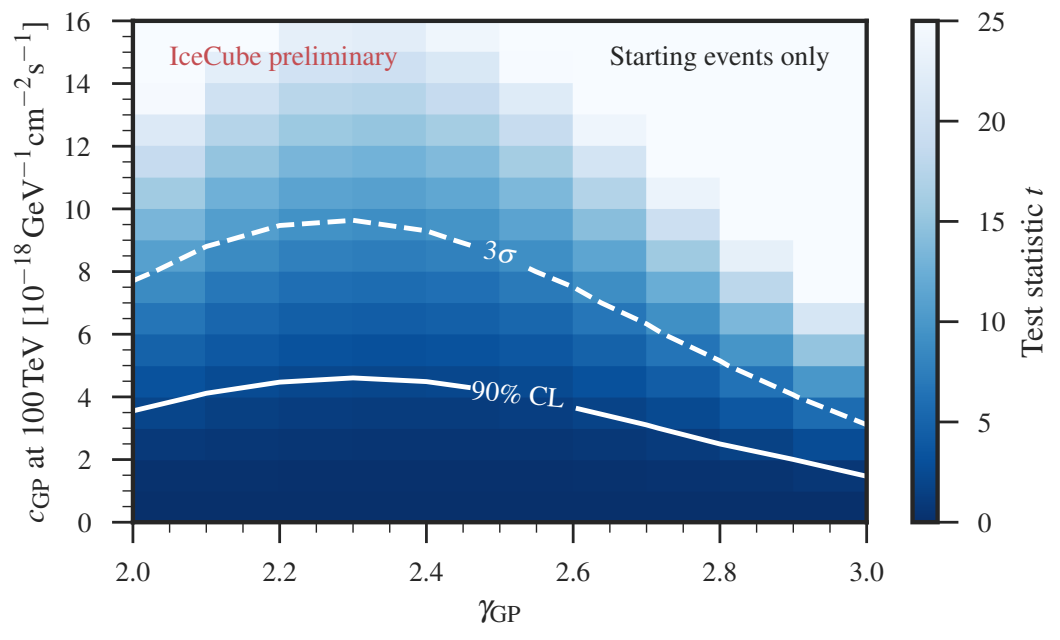

Figure 4: Sensitivity and $3 \sigma$ discovery potential differential neutrino flux $(v+\bar{v})$ per flavor assuming FermiLAT benchmark models of different Galactic spectral indices; color-coded is the underlying test statistic.

Figure 4 shows the sensitivity and $3 \sigma$ discovery potential for the Fermi-LAT benchmark model. At the reference Galactic spectral index of 2.5, the sensitivity on the flux normalization is $4.2 \times 10^{-18} \mathrm{GeV}^{-1} \mathrm{~cm}^{-2} \mathrm{~s}^{-1}$, which is of the same order of magnitude but worse than the values obtained in [2]. We expect the sensitivity to improve significantly once the through-going muon track selection is included in this analysis, because they will help to remove the degeneracy between the fitted extra-galactic and Galactic neutrino fluxes. For the KRA- $\gamma$ model with a high-energy cosmic-ray cutoff of $50 \mathrm{PeV}$, we obtain 0.7 and 1.6 times the model prediction for sensitivity and $3 \sigma$ discovery potential, respectively; compare Fig. 5 . The sensitivity is slightly better than the one presented in [2]. We benefit from the concentration of the KRA- $\gamma$ model towards the inner Galaxy and the Galactic Center; thus, more neutrinos are expected to arrive from the southern sky.

\section{Conclusions}

We presented the first steps towards a combined Galactic plane neutrino search using multiple IceCube event selections that follows the assumption that IceCube is measuring the superposition 


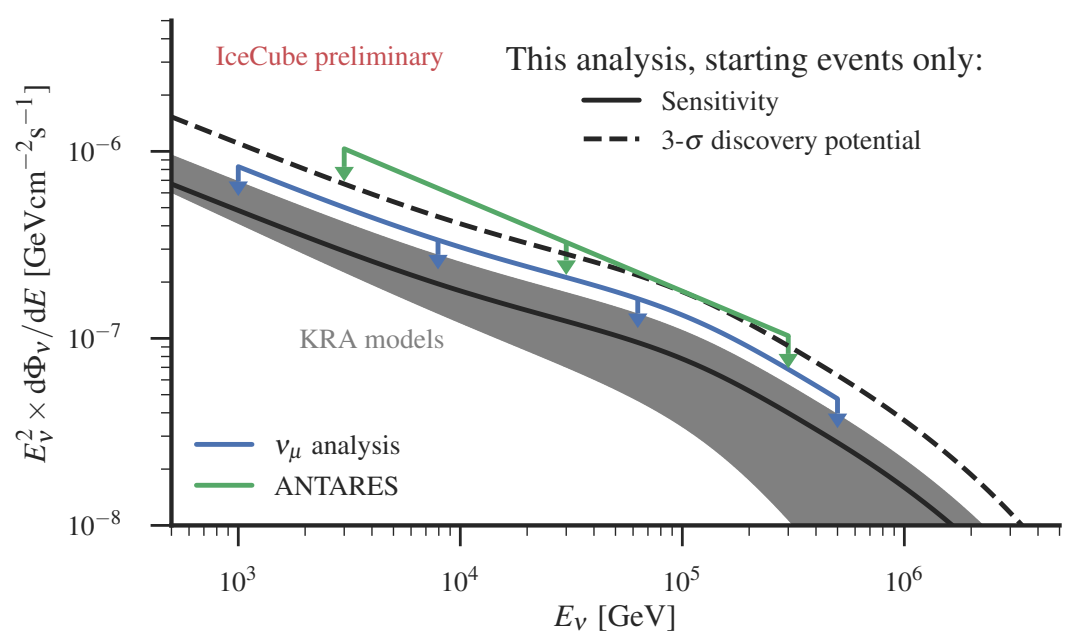

Figure 5: Sensitivity and $3 \sigma$ discovery potential differential all-flavor neutrino flux assuming the KRA- $\gamma$ model with a cosmic-ray high-energy cutoff of $50 \mathrm{PeV}$ and a neutrino flavor ratio of 1:1:1, compared to the IceCube [2] and ANTARES [13] upper limits. The gray band shows the envelope of the different realizations of the KRA model presented in [7].

of a hard isotropic neutrino flux of extra-galactic origin and a softer Galactic neutrino flux. Using six years of starting events, we obtain preliminary sensitivities for the Fermi-LAT and KRA- $\gamma$ Galactic plane models comparable to the search with through-going muon tracks in the northern sky. The next steps include the incorporation of detector-related systematic uncertainties, including a refined estimation of the atmospheric neutrino self-veto probabilities, and through-going muon tracks into the analysis.

\section{References}

[1] IceCube Collaboration, PoS (ICRC2017) 981 (these proceedings).

[2] IceCube Collaboration, PoS (ICRC2017) 1011 (these proceedings).

[3] T. K. Gaisser, Astropart. Phys. 35 (2012) 801-806.

[4] M. Honda et al., Phys. Rev. D83 (2011) 123001.

[5] R. Enberg et al., Phys. Rev. D78 (2008) 043005.

[6] Fermi-LAT Collaboration, M. Ackermann et al., Astrophys. J. 750 (2012) 3.

[7] D. Gaggero et al., POS ( ICRC2 015$) 489$ (2016).

[8] IceCube Collaboration, PoS ( ICRC2017) 976 (these proceedings).

[9] IceCube Collaboration, M. G. Aartsen et al., Phys. Rev. D91 (2015) 022001.

[10] T. K. Gaisser et al., Phys. Rev. D90 (2014) 023009.

[11] IceCube Collaboration, M. G. Aartsen et al., Astrophys. J. 833 (2016) 3.

[12] G. Cowan et al., Eur. Phys. J. C71 (2011) 1554.

[13] ANTARES Collaboration, S. Adrian-Martinez et al., Phys. Lett. B760 (2016) 143-148. 